\title{
Erratum
}

\section{Identifying a potential lion Panthera leo stronghold in Queen Elizabeth National Park, Uganda, and Parc National des Virunga, Democratic Republic of Congo-Erratum}

\author{
Adrian Treves, Andrew J. Plumptre, Luke T. B. Hunter and Joel Ziwa
}

doi:10.1017/So03060530700124X, Published by Cambridge University Press, 28 Jan 2009

Equation 3 of this paper contained a mistake that was replicated in Table 2. The corrections are reproduced below.

$L=(0.002+0.003 P) / 129 \quad($ Eq. 3)

TABLE 2 Observed prey numbers, prey biomass density and lion abundance, and estimates of lion abundance predicted from prey numbers using equations 2 and 3 (see text for further details) in Queen Elizabeth National Park complex, Uganda, in 1999 and 2004, and the adjoining Parc National des Virunga, DRC, in 2003 and 2006 (Fig. 1).

\begin{tabular}{|c|c|c|c|c|c|c|}
\hline \multicolumn{3}{|l|}{ Observed } & \multicolumn{4}{|c|}{ Lion abundance predicted from prey } \\
\hline \multirow[b]{2}{*}{$\begin{array}{l}\text { Prey numbers } \\
(\min -\max )\end{array}$} & \multirow[b]{2}{*}{$\begin{array}{l}\text { Prey biomass } \\
\text { density kg km } \\
(\text { min-max })\end{array}$} & \multirow[b]{2}{*}{ Lion numbers } & \multicolumn{2}{|l|}{ Equation 2} & \multicolumn{2}{|l|}{ Equation 3} \\
\hline & & & $\begin{array}{l}\text { Total } \\
(\min -\max )\end{array}$ & $\begin{array}{l}\text { Density, } \mathrm{km}^{-2} \\
(\min -\max )\end{array}$ & $\begin{array}{l}\text { Total } \\
(\min -\max )\end{array}$ & $\begin{array}{l}\text { Density, } \mathrm{km}^{-2} \\
(\min -\max )\end{array}$ \\
\hline \multicolumn{7}{|c|}{ Queen Elizabeth National Park, $1999\left(992 \mathrm{~km}^{2}\right.$; Dricuru, 1999) ${ }^{1}$} \\
\hline 19,126 & 4,672 & $105-116^{2}$ & 77.9 & 0.08 & 107.8 & 0.11 \\
\hline \multicolumn{7}{|c|}{ Queen Elizabeth National Park, $2004\left(641.9 \mathrm{~km}^{2}\right.$; JZ, unpubl. data) ${ }^{1}$} \\
\hline 13,223 & 5,692 & $88,59^{3}$ & 59.7 & 0.09 & 85.0 & 0.13 \\
\hline \multicolumn{7}{|c|}{ Parc National des Virunga, $2003\left(3,750\right.$ km$^{2}$; Mushenzi et al., 2003) } \\
\hline $\begin{array}{l}16,765 \\
(10,640-22,890)\end{array}$ & $\begin{array}{l}838 \\
(630-1,046)\end{array}$ & Unknown & $\begin{array}{l}70.9 \\
(51.0-88.8)\end{array}$ & $\begin{array}{l}0.02 \\
(0.01-0.02)\end{array}$ & $\begin{array}{l}73.1 \\
(55.0-91.3)\end{array}$ & $\begin{array}{l}0.02 \\
(0.01-0.02)\end{array}$ \\
\hline \multicolumn{7}{|c|}{ Parc National des Virunga, $2006\left(2,720.4 \mathrm{~km}^{2}\right.$; A. Plumptre et al., unpubl. data) } \\
\hline $\begin{array}{l}20,333 \\
(14,874-25,792)\end{array}$ & $\begin{array}{l}1,547 \\
(731-2,363)\end{array}$ & Unknown & $\begin{array}{l}81.5 \\
(65.0-96.9)\end{array}$ & $\begin{array}{l}0.03 \\
(0.02-0.04)\end{array}$ & $\begin{array}{l}97.9 \\
(46.3-149.5)\end{array}$ & $\begin{array}{l}0.04 \\
(0.02-0.06)\end{array}$ \\
\hline
\end{tabular}

${ }^{1}$ We assumed uniform distribution of prey from Table 1

${ }^{2}$ Dricuru (1999) total count, less 4 loners and 7 that died during the study

${ }^{3} \mathrm{JZ}$ (unpubl. data) conducted two surveys of the same area, in 2005 and 2007

\section{Reference}

Treves, A., Plumptre, A.J., Hunter, L.T.B. \& Ziwa, J. (2009) Identifying a potential lion Panthera leo stronghold in Queen Elizabeth National Park, Uganda, and Parc National des Virunga, Democratic Republic of Congo. Oryx, 43, 60-66. 